\title{
Generalized Hyers-Ulam Stability of a Mixed Type Functional Equation
}

\author{
Yang-Hi Lee ${ }^{1}$ and Soon-Mo Jung ${ }^{2}$ \\ ${ }^{1}$ Department of Mathematics Education, Gongju National University of Education, Gongju 314-711, Republic of Korea \\ ${ }^{2}$ Mathematics Section, College of Science and Technology, Hongik University, Sejong 339-701, Republic of Korea \\ Correspondence should be addressed to Soon-Mo Jung; smjung@hongik.ac.kr
}

Received 20 April 2013; Accepted 28 May 2013

Academic Editor: Bing Xu

Copyright (c) 2013 Y.-H. Lee and S.-M. Jung. This is an open access article distributed under the Creative Commons Attribution License, which permits unrestricted use, distribution, and reproduction in any medium, provided the original work is properly cited.

We investigate the stability of a functional equation $f(x+y+z)+f(x-y+z)+f(x+y-z)+f(-x+y+z)=3 f(x)+f(-x)+$ $3 f(y)+f(-y)+3 f(z)+f(-z)$ by applying the direct method in the sense of Hyers and Ulam.

\section{Introduction}

In 1940, Ulam [1] gave a wide ranging talk before the mathematics club of the University of Wisconsin in which he discussed a number of important unsolved problems. Among those was the question concerning the stability of group homomorphisms:

Let $G_{1}$ be a group and let $G_{2}$ be a metric group with the metric $d(\cdot, \cdot)$. Given $\varepsilon>0$, does there exist a $\delta>$ 0 such that if a function $h: G_{1} \rightarrow G_{2}$ satisfies the inequality $d(h(x y), h(x) h(y))<\delta$ for all $x, y \in G_{1}$, then there exists a homomorphism $H: G_{1} \rightarrow G_{2}$ with $d(h(x), H(x))<\varepsilon$ for all $x \in G_{1}$ ?

The Ulam's problem for the Cauchy additive functional equation was solved by Hyers under the assumption that $G_{1}$ and $G_{2}$ are Banach spaces. Indeed, Hyers [2] proved that every solution of the inequality $\|f(x+y)-f(x)-f(y)\| \leq \varepsilon$ (for all $x$ and $y$ ) can be approximated by an additive function. In this case, the Cauchy additive functional equation, $f(x+y)=$ $f(x)+f(y)$, is said to satisfy the Hyers-Ulam stability.

Thereafter, Rassias [3] attempted to weaken the condition for the bound of norm of the Cauchy difference as follows:

$$
\|f(x+y)-f(x)-f(y)\| \leq \varepsilon\left(\|x\|^{p}+\|y\|^{p}\right),
$$

and he proved that Hyers' theorem is also true for this case. Indeed, Rassias proved the generalized Hyers-Ulam stability (or the Hyers-Ulam-Rassias stability) of the Cauchy additive functional equation between Banach spaces. We here remark that a paper of Aoki [4] was published concerning the generalized Hyers-Ulam stability of the Cauchy functional equation earlier than Rassias' paper.

The stability concept that was introduced by Rassias' theorem provided a large influence to a number of mathematicians to develop the notion of what is known today with the term generalized Hyers-Ulam stability of functional equations. Since then, the stability problems of several functional equations have been extensively investigated by several mathematicians (e.g., see [5-10] and the references therein).

Almost all subsequent proofs in this very active area have used the Hyers' method presented in [2]. Namely, starting from the given mapping $f$ that approximately satisfies a given functional equation, a solution $F$ of the functional equation is explicitly constructed by using the formula:

$$
F(x):=\lim _{n \rightarrow \infty} \frac{1}{2^{n}} f\left(2^{n} x\right) \quad \text { or } \quad F(x):=\lim _{n \rightarrow \infty} 2^{n} f\left(\frac{x}{2^{n}}\right)
$$

which approximates the mapping $f$. This method of Hyers is called the direct method.

We remark that another method for proving the HyersUlam stability of various functional equations was introduced by Baker [11], which is called the fixed-point method. 
This method is very powerful technique of proving the stability of functional equations (see $[12,13]$ ).

Now we consider the following functional equation:

$$
\begin{aligned}
f(x+ & y+z)+f(x-y+z) \\
& +f(x+y-z)+f(-x+y+z) \\
= & 3 f(x)+f(-x)+3 f(y) \\
& +f(-y)+3 f(z)+f(-z),
\end{aligned}
$$

which is called the mixed type functional equation. The mapping $f(x)=a x^{2}+b x$ is a solution of this functional equation, where $a, b$ are real constants. Every solution of (3) will be called a quadratic-additive mapping.

In 1998, Jung [14] proved the stability of (3) by decomposing $f$ into the odd and even parts. In his proof, using the direct method, an additive mapping $A$ and a quadratic mapping $Q$ are separately constructed from the odd and even parts of $f$, and then $A$ and $Q$ are combined to provide a quadratic-additive mapping $F$ which is close to $f$.

In this paper, we will prove the generalized Hyers-Ulam stability of (3) by making use of the direct method. In particular, we will approximate the given mapping $f$ by a solution $F$ of (3) without decomposing $f$ into its odd and even parts, while in the Jung's paper [14] the mapping $f$ was decomposed into the odd and even parts, and each of them was separately approximated by the corresponding part of a solution $F$ of (3).

\section{Main Results}

Throughout this paper, let $X$ be a (real or complex) normed space and $Y$ a Banach space. For an arbitrary $p \in \mathbb{R}$, we define $s:=\operatorname{sign}(2-p)$ and $t:=\operatorname{sign}(1-p)$.

For a given mapping $f: X \rightarrow Y$, we use the following abbreviations:

$$
\begin{aligned}
f_{o}(x):= & \frac{f(x)-f(-x)}{2}, \\
f_{e}(x):= & \frac{f(x)+f(-x)}{2}, \\
J_{n} f(x):= & \frac{9^{-s n}}{2}\left(f\left(3^{s n} x\right)+f\left(-3^{s n} x\right)\right) \\
& +\frac{3^{-t n}}{2}\left(f\left(3^{t n} x\right)-f\left(-3^{t n} x\right)\right), \\
A f(x, y):= & f(x+y)-f(x)-f(y), \\
Q f(x, y):= & f(x+y)+f(x-y)-2 f(x)-2 f(y), \\
D f(x, y, z):= & f(x+y+z)+f(x-y+z) \\
& +f(x+y-z)+f(-x+y+z) \\
& -3 f(x)-f(-x)-3 f(y)
\end{aligned}
$$

$$
-f(-y)-3 f(z)-f(-z)
$$

for all $x, y, z \in X$.

As we stated in the previous section, $f$ is called a quadratic-additive mapping provided that $f$ satisfies the functional equation $D f(x, y, z)=0$ for all $x, y, z \in X$.

Proposition 1. A mapping $f: X \rightarrow Y$ is a solution of (3) if and only if $f_{e}$ is a quadratic mapping and $f_{o}$ is an additive mapping.

Proof. Assume that $f: X \rightarrow Y$ is a solution of (3). Then we have

$$
\begin{gathered}
Q f_{e}(x, y, z)=\frac{D f_{e}(x, y, 0)}{2}=0, \\
A f_{o}(x, y, z)=\frac{D f_{o}(x, y, 0)}{2}=0
\end{gathered}
$$

for all $x, y, z \in X$, that is, $f_{e}$ is a quadratic mapping and $f_{o}$ is an additive mapping.

Conversely, assume that $f_{e}$ is a quadratic mapping and $f_{o}$ is an additive mapping. Then we get

$$
\begin{aligned}
D f(x, y, z)= & D f_{e}(x, y, z)+D f_{o}(x, y, z) \\
= & Q f_{e}(x+y, z)+Q f_{e}(x-y, z) \\
& +2 Q f_{e}(x, y)+A f_{o}(x+y, z) \\
& +A f_{o}(x+y,-z)+A f_{o}(x-y, z) \\
& +A f_{o}(-x+y, z)+2 A f_{o}(x, y) \\
= & 0
\end{aligned}
$$

for all $x, y, z \in X$; that is, $f$ is a solution of (3).

We first prove the following lemma.

Lemma 2. If a mapping $f: X \rightarrow Y$ satisfies $D f(x, y, z)=0$ for all $x, y, z \in X \backslash\{0\}$ and $f(0)=0$, then $f$ is a quadraticadditive mapping.

Proof. Using the hypothesis, we have

$$
\begin{aligned}
f(2 x)-3 f(x)-f(-x) & \\
=\frac{11}{112}( & D f(4 x, 3 x, x)-D f(4 x, 2 x, 2 x) \\
- & D f(2 x, 2 x, 2 x)+2 D f(2 x, x, x) \\
+ & 3 D f(x, x, x)+D f(-x,-x,-x)) \\
-\frac{3}{112}( & D f(-4 x,-3 x,-x)-D f(-4 x,-2 x,-2 x) \\
& -D f(-2 x,-2 x,-2 x)+2 D f(-2 x,-x,-x) \\
+ & 3 D f(-x,-x,-x)+D f(x, x, x))
\end{aligned}
$$$$
=0
$$ 
for all $x \in X \backslash\{0\}$. Furthermore, by the last equality, we get

$$
\begin{aligned}
D f(x, y, 0)= & D f\left(x, \frac{y}{2}, \frac{y}{2}\right)+D f\left(y, \frac{x}{2}, \frac{x}{2}\right) \\
& -2 f(x)+6 f\left(\frac{x}{2}\right)+2 f\left(-\frac{x}{2}\right) \\
& -2 f(y)+6 f\left(\frac{y}{2}\right)+2 f\left(-\frac{y}{2}\right) \\
& =0
\end{aligned}
$$

for all $x, y \in X \backslash\{0\}$. Since $D f(x, y, z)$ is invariant with respect to the permutation of $(x, y, z)$, it holds that $D f(0, y, z)=$ $D f(x, 0, z)=0$ for all $x, y, z \in X \backslash\{0\}$. It is also easy to show that $D f(x, 0,0)=0, D f(0, y, 0)=0, D f(0,0, z)=0$, and $D f(0,0,0)=0$ for all $x, y, z \in X \backslash\{0\}$ as we desired.

In the following theorem, we can prove the generalized Hyers-Ulam stability of the functional equation (3) by making use of the direct method.

Theorem 3. If a mapping $f: X \rightarrow Y$ satisfies $f(0)=0$ and

$$
\|D f(x, y, z)\| \leq\|x\|^{p}+\|y\|^{p}+\|z\|^{p}
$$

for all $x, y, z \in X \backslash\{0\}$ with a real constant $p \notin\{1,2\}$, then there exists a unique quadratic-additive mapping $F: X \rightarrow Y$ such that

$$
\begin{aligned}
& \|f(x)-F(x)\| \\
& \quad \leq \begin{cases}\frac{3\|x\|^{p}}{3^{p}-9} & (\text { for } p>2), \\
\left(\frac{3}{9-3^{p}}+\frac{3}{3^{p}-3}\right)\|x\|^{p} & (\text { for } 1<p<2), \\
\frac{3\|x\|^{p}}{3-3^{p}} & (\text { for } 0 \leq p<1)\end{cases}
\end{aligned}
$$

for all $x \in X \backslash\{0\}$. Moreover, if $p<0$, then $f$ itself is a quadratic-additive mapping.

Proof. Let us define $\tau_{s, n}:=s(n+1 / 2)-1 / 2$, where $s \in\{-1,1\}$. From the definitions of $J_{n} f(x)$ and $D f(x, y, z)$, we have

$$
\begin{aligned}
J_{n} f(x)-J_{n+1} f(x) & \\
=-\frac{1}{2}\left(9^{\tau_{-s, n}}(\right. & D f\left(3^{\tau_{s, n}} x, 3^{\tau_{s, n}} x, 3^{\tau_{s, n}} x\right) \\
+ & \left.D f\left(-3^{\tau_{s, n}} x,-3^{\tau_{s, n}} x,-3^{\tau_{s, n}} x\right)\right) s \\
+3^{\tau_{-t, n}}( & D f\left(3^{\tau_{t, n}} x, 3^{\tau_{t, n}} x, 3^{\tau_{t, n}} x\right) \\
& \left.\left.-D f\left(-3^{\tau_{t, n}} x,-3^{\tau_{t, n}} x,-3^{\tau_{t, n}} x\right)\right) t\right)
\end{aligned}
$$

for all $x \in X \backslash\{0\}$ and $n \in \mathbb{N}_{0}$. It follows from (9) and (11) that

$$
\begin{aligned}
& \left\|J_{n} f(x)-J_{n+m} f(x)\right\| \\
& =\sum_{j=n}^{n+m-1}\left\|J_{j} f(x)-J_{j+1} f(x)\right\| \\
& \leq \frac{1}{2} \sum_{j=n}^{n+m-1}\left(\| 9^{\tau_{-s, j}} D f\left(3^{\tau_{s, j}} x, 3^{\tau_{s, j}} x, 3^{\tau_{s, j}} x\right) s\right. \\
& +3^{\tau_{-t, j}} D f\left(3^{\tau_{t, j}} x, 3^{\tau_{t, j}} x, 3^{\tau_{t, j}} x\right) t \| \\
& +\| 9^{\tau_{-s, j}} D f\left(-3^{\tau_{s, j}} x,-3^{\tau_{s, j}} x,-3^{\tau_{s, j}} x\right) s \\
& \left.-3^{\tau_{-t, j}} D f\left(-3^{\tau_{t, j}} x,-3^{\tau_{t, j}} x,-3^{\tau_{t, j}} x\right) t \|\right)
\end{aligned}
$$$$
\leq \begin{cases}\sum_{j=n}^{n+m-1} 3^{-j}\left\|3^{j} x\right\|^{p} & (\text { for } p<1), \\ \sum_{j=n}^{n+m-1}\left(3^{-2 j-1}\left\|3^{j} x\right\|^{p}+3^{j+1}\left\|3^{-j-1} x\right\|^{p}\right) & (\text { for } 1<p<2), \\ \sum_{j=n}^{n+m-1} 3^{2 j+1}\left\|3^{-j-1} x\right\|^{p} & (\text { for } p>2)\end{cases}
$$$$
\leq \begin{cases}\frac{3^{n p}\|x\|^{p}}{3^{n-1}\left(3-3^{p}\right)} & (\text { for } p<1), \\ \frac{3^{n p}\|x\|^{p}}{3^{2 n-1}\left(9-3^{p}\right)}+\frac{3^{n+1}\|x\|^{p}}{3^{n p}\left(3^{p}-3\right)} & (\text { for } 1<p<2), \\ \frac{3^{2 n+1}\|x\|^{p}}{3^{n p}\left(3^{p}-9\right)} & (\text { for } p>2)\end{cases}
$$

for all $x \in X \backslash\{0\}$. So, it is easy to show that the sequence $\left\{J_{n} f(x)\right\}$ is a Cauchy sequence for all $x \in X \backslash\{0\}$.

Since $Y$ is complete and $f(0)=0$, the sequence $\left\{J_{n} f(x)\right\}$ converges for all $x \in X$. Hence, we can define a mapping $F$ : $X \rightarrow Y$ by

$$
F(x):=\lim _{n \rightarrow \infty} J_{n} f(x)
$$

for all $x \in X$. Moreover, if we put $n=0$ and let $m \rightarrow \infty$ in (12), we obtain the inequality (10).

From the definition of $F$, we get

$$
\begin{aligned}
& D F(x, y, z) \\
& =\lim _{n \rightarrow \infty} \frac{9^{-s n}}{2}\left(D f\left(3^{s n} x, 3^{s n} y, 3^{s n} z\right)\right. \\
& \left.+D f\left(-3^{s n} x,-3^{s n} y,-3^{s n} z\right)\right) \\
& \quad+\lim _{n \rightarrow \infty} \frac{3^{-t n}}{2}\left(D f\left(3^{t n} x, 3^{t n} y, 3^{t n} z\right)\right. \\
& \left.\quad-D f\left(-3^{t n} x,-3^{t n} y,-3^{t n} z\right)\right) \\
& \leq \lim _{n \rightarrow \infty}\left(3^{-s(2-p) n}+3^{-t n(1-p)}\right)\left(\|x\|^{p}+\|y\|^{p}+\|z\|^{p}\right) \\
& =0
\end{aligned}
$$


for all $x, y, z \in X \backslash\{0\}$. By Lemma 2, $F$ is a quadratic-additive mapping.

Now, we will show that $F$ is uniquely determined. Let $F^{\prime}$ : $X \rightarrow Y$ be another quadratic-additive mapping satisfying (10). It is easy to show that $F^{\prime}(0)=0$ for all quadratic-additive mapping $F^{\prime}$. It follows from (11) that

$$
\begin{gathered}
F^{\prime}(x)-J_{n} F^{\prime}(x) \\
=\sum_{j=0}^{n-1}\left(J_{j} F^{\prime}(x)-J_{j+1} F^{\prime}(x)\right) \\
=-\frac{1}{2} \sum_{j=0}^{n-1}\left(9 ^ { \tau _ { - s , j } } \left(D F^{\prime}\left(3^{\tau_{s, j}} x, 3^{\tau_{s, j}} x, 3^{\tau_{s, j}} x\right)\right.\right. \\
\left.+D F^{\prime}\left(-3^{\tau_{s, j}} x,-3^{\tau_{s, j}} x,-3^{\tau_{s, j}} x\right)\right) s \\
+3^{\tau_{-t, j}}\left(D F^{\prime}\left(3^{\tau_{t, j}} x, 3^{\tau_{t, j}} x, 3^{\tau_{t, j}} x\right)\right. \\
\left.\left.-D F^{\prime}\left(-3^{\tau_{t, j}} x,-3^{\tau_{t, j}} x,-3^{\tau_{t, j}} x\right)\right) t\right)
\end{gathered}
$$$$
=0
$$

for all $n \in \mathbb{N}$ and $x \in X$. Since $F$ and $F^{\prime}$ are quadratic-additive, if we replace $x$ with $3^{n} x$ in (10), then we have

$$
\begin{aligned}
& \left\|F(x)-F^{\prime}(x)\right\| \\
& =\left\|J_{n} F(x)-J_{n} F^{\prime}(x)\right\| \\
& \leq \frac{9^{-s n}}{2}\left(\left\|(F-f)\left(3^{s n} x\right)\right\|+\left\|\left(f-F^{\prime}\right)\left(3^{s n} x\right)\right\|\right. \\
& \left.\quad+\left\|(F-f)\left(-3^{s n} x\right)\right\|+\left\|\left(f-F^{\prime}\right)\left(-3^{s n} x\right)\right\|\right) \\
& \quad+\frac{2^{-t n}}{2}\left(\left\|(F-f)\left(3^{t n} x\right)\right\|+\left\|\left(F^{\prime}-f\right)\left(3^{t n} x\right)\right\|\right. \\
& \left.\quad+\left\|(F-f)\left(-3^{t n} x\right)\right\|+\left\|\left(F^{\prime}-f\right)\left(-3^{t n} x\right)\right\|\right) \\
& \leq\left(\frac{6}{\left|9-3^{p}\right|}+\frac{6}{\left|3^{p}-3\right|}\right)\left(3^{-s n(2-p)}+3^{-\operatorname{tn}(1-p)}\right)\|x\|^{p}
\end{aligned}
$$

for all $x \in X \backslash\{0\}$ and $n \in \mathbb{N}$. Taking the limit in the above inequality as $n \rightarrow \infty$, we can conclude that $F(x)=F^{\prime}(x)$ for all $x \in X$, which proves the uniqueness of $F$.

Since

$$
\begin{aligned}
\| f(x) & -F(x) \| \\
\leq & \|D f((2 k-1) x, k x, k x)-D F((2 k-1) x, k x, k x)\| \\
& +\|F((4 k-1) x)-f((4 k-1) x)\| \\
& +\|f((2 k-1) x)-F((2 k-1) x)\| \\
& +\|f((1-2 k) x)-F((1-2 k) x)\| \\
& +2\|f(-k x)-F(-k x)\|
\end{aligned}
$$

$$
\begin{aligned}
& +6\|f(k x)-F(k x)\| \\
\leq & \left((2 k-1)^{p}+2 k^{p}+\frac{3\left((4 k-1)^{p}+2(2 k-1)^{p}+8 k^{p}\right)}{3-3^{p}}\right) \\
& \times\|x\|^{p}
\end{aligned}
$$

for all $x \in X \backslash\{0\}$ and $k \in \mathbb{N}$, if $p<0$, then we conclude that $f(x)=F(x)$ for all $x \in X \backslash\{0\}$ by letting $k \rightarrow \infty$ in the previous inequality. From the fact that $f(0)=0, f$ is a quadratic-additive mapping.

Theorem 4. If a mapping $f: X \rightarrow Y$ satisfies

$$
\|D f(x, y, z)\| \leq\|x\|^{p}+\|y\|^{p}+\|z\|^{p}
$$

for all $x, y, z \in X$ and for a nonnegative real constant $p \notin$ $\{1,2\}$, then there exists a unique quadratic-additive mapping $F: X \rightarrow Y$ such that

$$
\|f(x)-F(x)\|
$$

$$
\leq \begin{cases}\frac{\|x\|^{p}}{2^{p}-4} & (\text { for } p>2), \\ \left(\frac{1}{4-2^{p}}+\frac{1}{2^{p}-2}\right)\|x\|^{p} & (\text { for } 1<p<2), \\ \frac{\|x\|^{p}}{2-2^{p}} & (\text { for } 0<p<1), \\ \frac{25}{16} & (\text { for } p=0)\end{cases}
$$

for all $x \in X$.

Proof. Since

$$
\|f(0)\|=\left\|\frac{1}{8} D f(0,0,0)\right\| \leq \frac{3\|0\|^{p}}{8},
$$

we get $f(0)=0$ for $p \notin\{0,1,2\}$ and $\|f(0)\| \leq 3 / 8$ for $p=0$. From the definitions of $J_{n} f(x)$ and $D f(x, y, z)$, we have

$$
\begin{gathered}
J_{n} f(x)-J_{n+1} f(x) \\
=-\frac{1}{4}\left(4 ^ { \tau _ { - s , n } } \left(D f\left(2^{\tau_{s, n}} x, 2^{\tau_{s, n}} x, 0\right)\right.\right. \\
\left.+D f\left(-2^{\tau_{s, n}} x,-2^{\tau_{s, n}} x, 0\right)\right) s \\
+2^{\tau_{-t, n}}\left(D f\left(2^{\tau_{t, n}} x, 2^{\tau_{t, n}} x, 0\right)\right. \\
\left.\left.-D f\left(-2^{\tau_{t, n}} x,-2^{\tau_{t, n}} x, 0\right)\right) t\right) \\
+\frac{1}{2} 4^{\tau_{-s, n}} f(0)
\end{gathered}
$$

for all $x \in X$ and $n \in \mathbb{N}_{0}$, where $\tau_{s, n}$ is defined by $\tau_{s, n}=$ $s(n+1 / 2)-1 / 2$ and $s \in\{-1,1\}$. 
It follows from (18) and (21) that

$$
\begin{aligned}
& \left\|J_{n} f(x)-J_{n+m} f(x)\right\| \\
& =\sum_{j=n}^{n+m-1}\left\|J_{j} f(x)-J_{j+1} f(x)\right\| \\
& \leq \frac{1}{4} \sum_{j=n}^{n+m-1}\left(\| 4^{\tau_{-s, j}} D f\left(2^{\tau_{s, j}} x, 2^{\tau_{s, j}} x, 0\right) s\right. \\
& +2^{\tau_{-t, j}} D f\left(2^{\tau_{t, j}} x, 2^{\tau_{t, j}} x, 0\right) t \| \\
& +\| 4^{\tau_{-s, j}} D f\left(-2^{\tau_{s, j}} x,-2^{\tau_{s, j}} x, 0\right) s \\
& -2^{\tau_{-t, j}} D f\left(-2^{\tau_{t, j}} x,-2^{\tau_{t, j}} x, 0\right) t \| \\
& \left.+2 \cdot 4^{\tau_{-s, j}}\|f(0)\|\right) \\
& \sum_{j=n}^{n+m-1} \frac{3}{2^{j+2}}+\frac{3 \cdot 2^{-2 j-3}}{8} \quad(\text { for } p=0) \\
& \leq \begin{cases}\sum_{j=n}^{n+m-1} 2^{-j-1}\left\|2^{j} x\right\|^{p} & (\text { for } 0<p<1), \\
\sum_{j=n}^{n+m-1} 2^{-2 j-2}\left\|2^{j} x\right\|^{p}+2^{j}\left\|2^{-j-1} x\right\|^{p} & (\text { for } 1<p<2), \\
\sum_{j=n}^{n+m-1} 2^{2 j}\left\|2^{-j-1} x\right\|^{p} & (\text { for } p>2)\end{cases} \\
& \leq \begin{cases}\frac{3}{2^{n+1}}+\frac{1}{2^{2 n+4}} & (\text { for } p=0), \\
\frac{2^{n p}\|x\|^{p}}{2^{n}\left(2-2^{p}\right)} & (\text { for } 0<p<1), \\
\frac{2^{n p}\|x\|^{p}}{4^{n}\left(4-2^{p}\right)}+\frac{2^{n}\|x\|^{p}}{2^{n p}\left(2^{p}-2\right)} & (\text { for } 1<p<2), \\
\frac{4^{n}\|x\|^{p}}{2^{n p}\left(2^{p}-4\right)} & (\text { for } p>2)\end{cases}
\end{aligned}
$$

for all $x \in X$. So, it is easy to show that the sequence $\left\{J_{n} f(x)\right\}$ is a Cauchy sequence for all $x \in X$.

Since $Y$ is complete, the sequence $\left\{J_{n} f(x)\right\}$ converges for all $x \in X$. Hence, we can define a mapping $F: X \rightarrow Y$ by

$$
F(x):=\lim _{n \rightarrow \infty} J_{n} f(x)
$$

for all $x \in X$. Moreover, putting $n=0$ and letting $m \rightarrow \infty$ in (22), we get the inequality (19).

\section{Acknowledgment}

This research was supported by Basic Science Research Program through the National Research Foundation of Korea (NRF) funded by the Ministry of Education, Science and Technology (no. 2012R1A1A4A01002971).

\section{References}

[1] S. M. Ulam, Problems in Modern Mathematics, John Wiley \& Sons, New York, NY, USA, 1964.

[2] D. H. Hyers, "On the stability of the linear functional equation," Proceedings of the National Academy of Sciences of the United States of America, vol. 27, pp. 222-224, 1941.

[3] T. M. Rassias, "On the stability of the linear mapping in Banach spaces," Proceedings of the American Mathematical Society, vol. 72, no. 2, pp. 297-300, 1978.

[4] T. Aoki, "On the stability of the linear transformation in Banach spaces," Journal of the Mathematical Society of Japan, vol. 2, pp. 64-66, 1950.

[5] S. Czerwik, Functional Equations and Inequalities in Several Variables, World Scientific, Singapore, 2002.

[6] M. E. Gordji and M. Ramezani, "Erdös problem and quadratic equation," Annals of Functional Analysis, vol. 1, no. 2, pp. 64-67, 2010.

[7] D. H. Hyers, G. Isac, and T. M. Rassias, Stability of Functional Equations in Several Variables, Progress in Nonlinear Differential Equations and their Applications, 34, Birkhäuser, Basel, Switzerland, 1998.

[8] S.-M. Jung, "Hyers-Ulam-Rassias stability of Jensen's equation and its application," Proceedings of the American Mathematical Society, vol. 126, no. 11, pp. 3137-3143, 1998.

[9] S.-M. Jung, Hyers-Ulam-Rassias Stability of Functional Equations in Nonlinear Analysis, vol. 48 of Springer Optimization and Its Applications, Springer, New York, NY, USA, 2011.

[10] S. S. Zhang, R. Saadati, and G. Sadeghi, "Solution and stability of mixed type functional equation in non-Archimedean random normed spaces," Applied Mathematics and Mechanics (English Edition), vol. 32, no. 5, pp. 663-676, 2011.

[11] J. A. Baker, "The stability of certain functional equations," Proceedings of the American Mathematical Society, vol. 112, no. 3, pp. 729-732, 1991.

[12] K. Ciepliński, "Applications of fixed point theorems to the Hyers-Ulam stability of functional equations-a survey," Annals of Functional Analysis, vol. 3, no. 1, pp. 151-164, 2012.

[13] M. Mirzavaziri and M. S. Moslehian, "A fixed point approach to stability of a quadratic equation," Bulletin of the Brazilian Mathematical Society, vol. 37, no. 3, pp. 361-376, 2006.

[14] S.-M. Jung, "On the Hyers-Ulam stability of the functional equations that have the quadratic property," Journal of Mathematical Analysis and Applications, vol. 222, no. 1, pp. 126-137, 1998. 


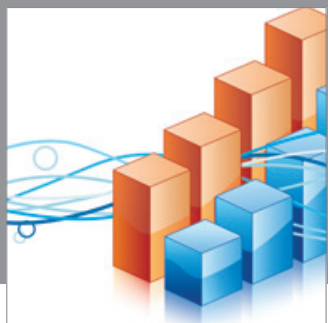

Advances in

Operations Research

mansans

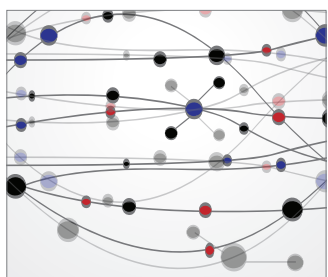

The Scientific World Journal
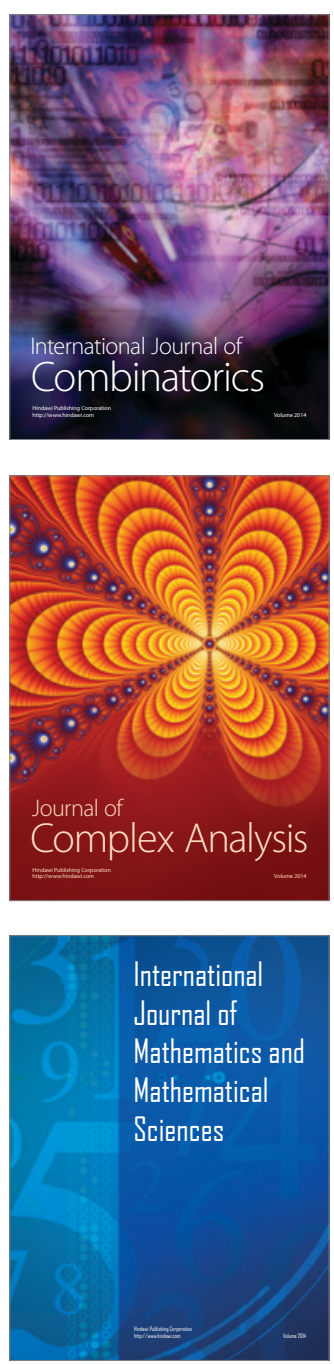
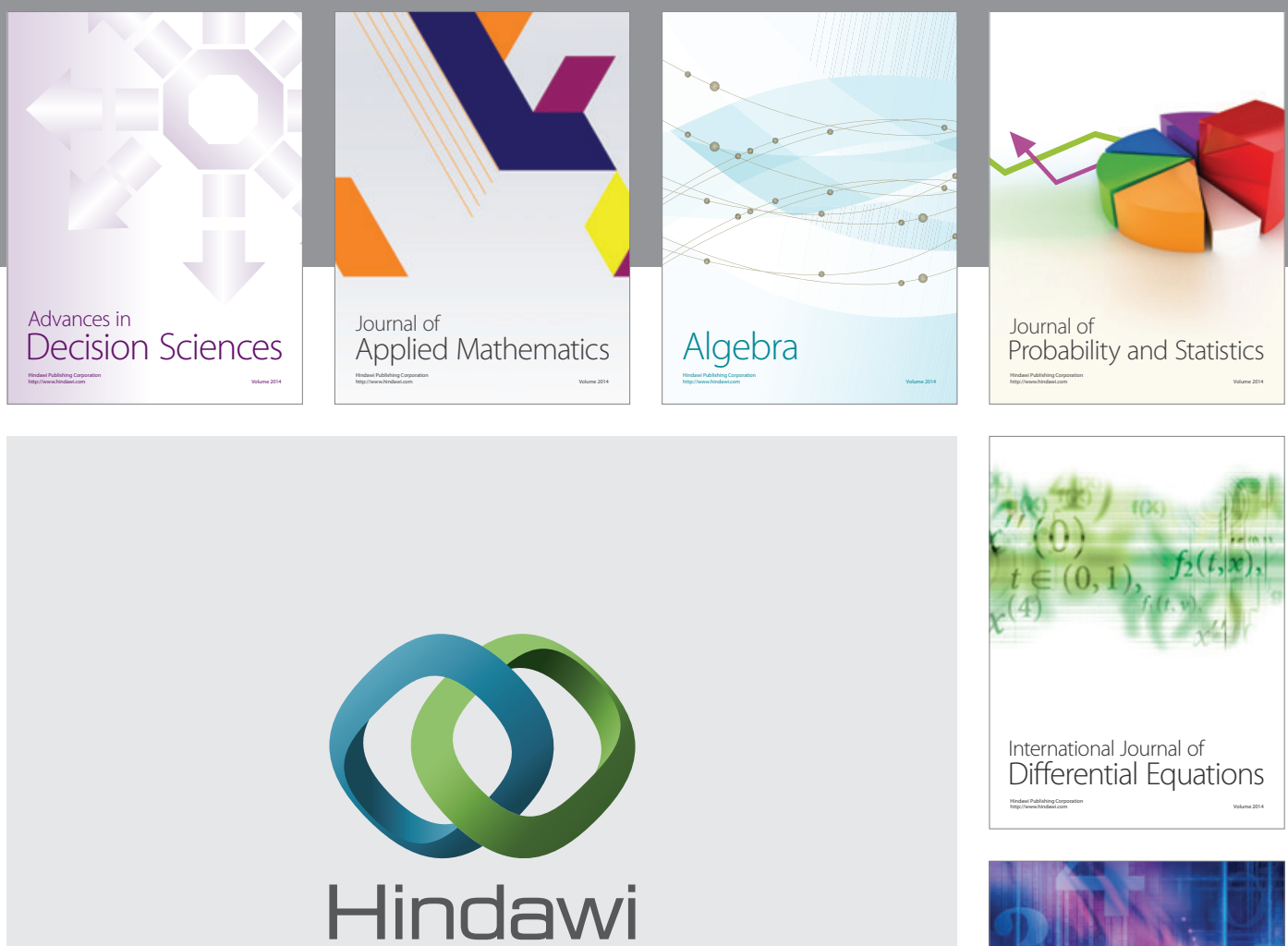

Submit your manuscripts at http://www.hindawi.com
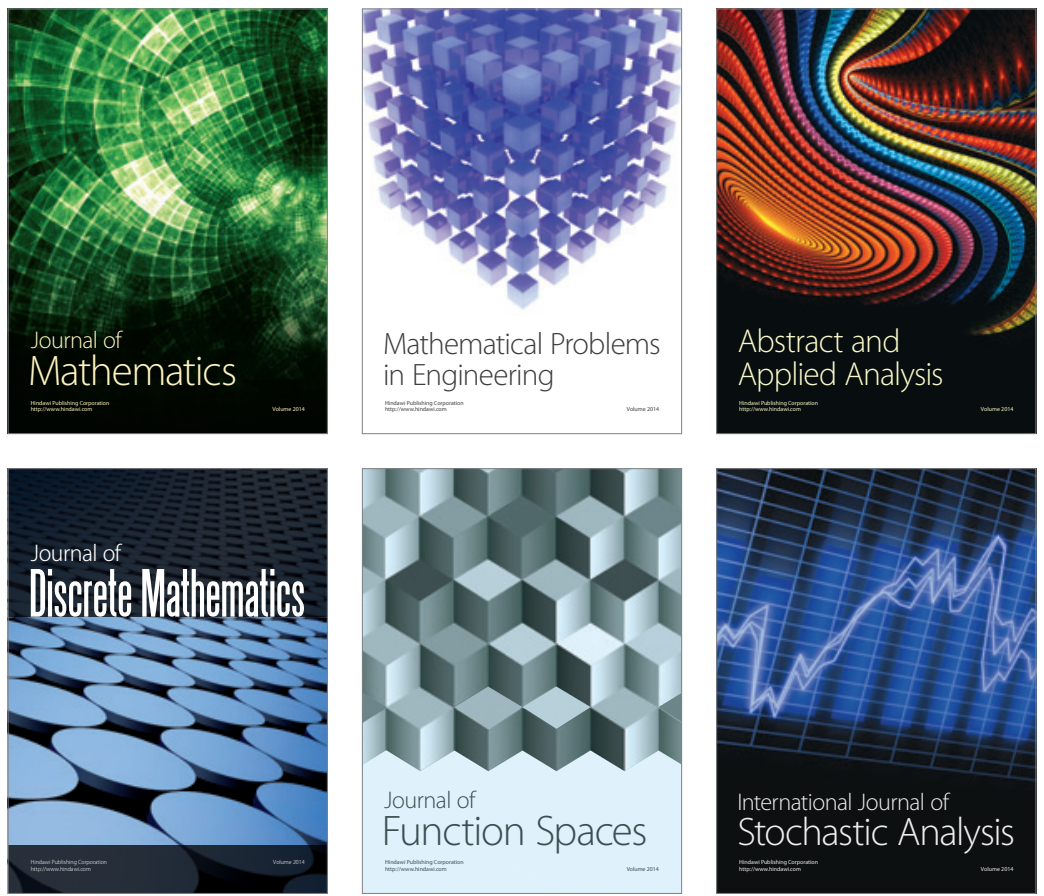

Journal of

Function Spaces

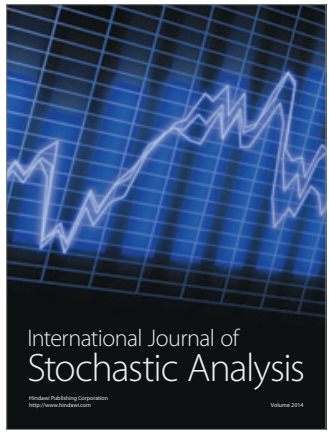

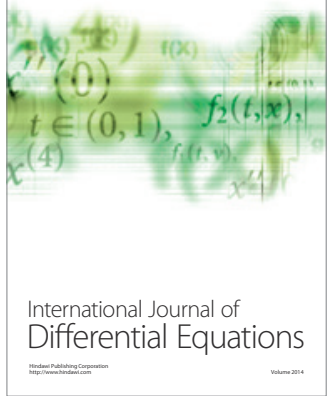
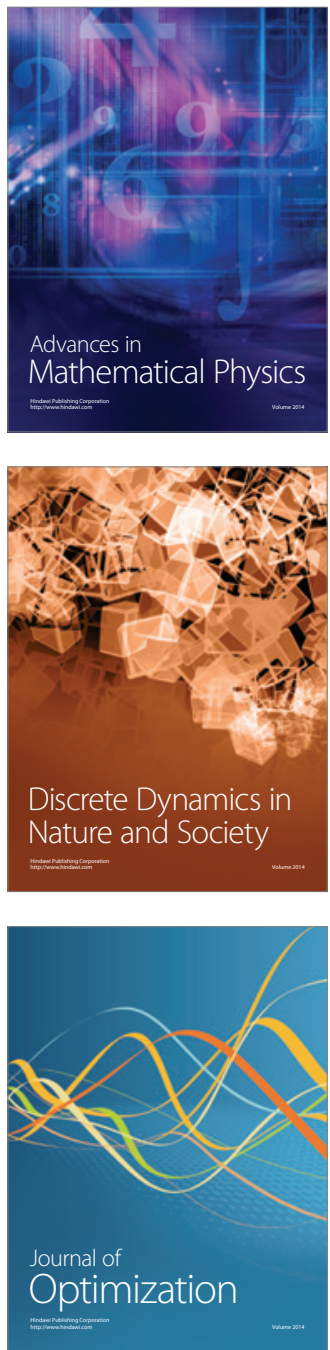